\title{
Methodology for the at-home collection of urine samples for prostate cancer detection
}

Martyn Webb', Kate Manley',2, Mireia Olivan', Ingrid Guldvik ${ }^{5,6}$, Malgorzata Palczynska ${ }^{2}$, Rachel Hurst', Shea P Connell', lan G Mills ${ }^{7}$, Daniel S Brewer ${ }^{1,3}$, Robert Mills ${ }^{2}$, Colin S Cooper ${ }^{1} \&$ Jeremy Clark ${ }^{\star, 1}$

\section{ABSTRACT}

Urine from patients with prostate cancer ( $\mathrm{PCa}$ ) contains gene transcripts that have been used for $\mathrm{PC}$ a diagnosis and prognosis. Historically, patient urine samples have been collected after a digital rectal examination of the prostate, which was thought necessary to boost the levels of prostatic secretions in the urine. We herein describe methodology that allows urine to be collected by patients at home and then posted to a laboratory for analysis. RNA yields and quality were comparable to those for post digital rectal examination urine, and there was improved sensitivity for the detection of TMPRSS2:ERG transcripts by RT-PCR. The At-Home collection protocol has opened up the potential to perform large-scale PCa studies without the inconvenience, cost, discomfort and expense of patients having to visit the clinic.

\section{METHOD SUMMARY}

The use of a commercial preservative allowed samples to be maintained at room temperature without loss of RNA quality. Harvest of cell-free RNA using a novel high-volume vacuum extraction method increased total RNA yields, improved the detection sensitivity of prostate-cancer-specific transcripts by RT-PCR, enabled extraction of RNA from historic frozen urine samples, and allowed the harvest of sRNA. Comparisons between digital rectal examination (DRE) and non-DRE urine RNA yields and RT-PCR expression levels have demonstrated that the collection of non-DRE urine by men at home is a viable and simple option.

\section{KEYWORDS}

biomarker cancer $\cdot$ diagnosis $\cdot$ home-screening - prognosis $\cdot$ prostate $\cdot$ urine

'Norwich Medical School, University of East Anglia, Norwich, UK; ${ }^{2}$ Norfolk \& Norwich University Hospitals NHS Foundation Trust, Norwich, UK; ${ }^{3}$ Earlham Institute, Norwich, UK; ${ }^{4}$ Group of Biomedical Research in Urology, Vall d'Hebron Research Institute \& Universitat Autònoma de Barcelona, Barcelona, Spain; ${ }^{5}$ Prostate Cancer Research Group, Centre for Molecular Medicine Norway, University of Oslo \& Oslo University Hospital, Oslo, Norway; ${ }^{6}$ Department of Tumour Biology, Institute for Cancer Research, Oslo University Hospital, Oslo, Norway; ${ }^{7}$ School of Medicine, Dentistry \& Biomedical Sciences, Institute for Health Sciences, Centre for Cancer Research \& Cell Biology, Queen's University Belfast, Belfast, UK; *Author for correspondence: Jeremy.clark@uea.ac.uk

BioTechniques 68: 65-71 (February 2020) 10.2144/btn-2019-0092
Prostate cancer ( $\mathrm{PCa})$ is present in nearly half of all men over 60 years [1]; however, only a very small proportion of these men will die of PCa [2]. Determining which men have disease that requires treatment is an ongoing clinical problem. Furthermore, the majority of PCa cases are multifocal [3-5] and tumors with different Gleason patterns can exist in individual prostates [3], adding complexity to disease monitoring and assessment. The zones of the prostate where tumors are found constantly produce secretions that naturally flow into the urethra [6]. These secretions carry cancer cells and cell-free RNA (cfRNA) contained within extracellular vesicles [7] that are flushed out of the body upon urination. RNA can be harvested from urine and examined for the presence of PCa transcripts and prognostic markers. Recent successes in this field include three studies, two carried out on whole urine and one on cfRNA [8-10]. All three papers generated similar AUROC (area under the receiver operating characteristics) curves of 0.73 to 0.78 for the detection of significant cancer (cancer with a pathological Gleason score of $\geq 7$ ). The study by Connell et al. [8] also identified predictors of disease progression in active surveillance patients up to 5 years after a single urine sample (hazard ratio: $8.23 ; 95 \% \mathrm{Cl}: 3.26-20.81 ; p<0.001$ ). These studies and the PCA3 test all measure transcripts in urine collected after a digital rectal examination (DRE) of the prostate, which boosts levels of prostate transcripts in the urine [11]. A DRE involves a clinician inserting a finger into the rectum and firmly stroking the entire posterior side of the prostate. There are a number of issues with the DRE. There is variation in the size and position of the prostate, which may not be easily accessible [12]. A DRE depresses only the posterior side of the prostate, thus increasing the detection of cancers on that side of the prostate relative to anterior cancers. Also, a DRE requires a visit to a clinic, and DREs are unpopular with patients [13]. McKiernan et al. [14] recently demonstrated that cfRNA extracted from non-DRE urine collected in the clinic could be used to detect significant PCa with a comparable AUROC of 0.77 [15]. However, we have found that non-DRE urine collected in the clinic is highly variable and can have very poor yields of RNA [8]. We hypothesize that this is due to men urinating immediately prior to seeing a doctor and thus depleting prostate secretions from the urethra.

Here we describe an at-home collection system for acquiring urine from the first micturition of the day for diagnostic and prognostic use. This at-home collection protocol will allow the utility of urine to be explored more widely and easily, will minimize costly and stressful visits to the clinic, and could lead to the implementation of an early intervention test for PCa akin to that used for colorectal cancer [16]. This method also has the potential to be used in a wider arena to detect other urological malignancies present in bladder and kidney tissues.

\section{MATERIALS \& METHODS}

Manufacturer and catalog number information for all items used in this study are presented in the Supplementary data. 
Urine sample collection

Urine samples (first $\leq 30 \mathrm{ml}$ voided) were collected from men attending urology clinics at the Norfolk and Norwich University Hospital. Samples were either taken before biopsy or collected more than 3 months after biopsy. Ethical approval was obtained from the East of England Research Ethics Committee (IRAS project ID 96199, REC reference 12/EE/0058). A pre-addressed, first-class postage-prepaid, leakproof SafeBox container was included in all of the at-home kits. In this study, all samples were received from donors within 3 days of collection.

\section{RNA extraction}

Each sample was centrifuged at $2500 \times g$ for $5 \mathrm{~min}$. Supernatant was filtered through a $0.8-\mathrm{mm}$ filter, and the cell pellet was stored in $1 \mathrm{ml}$ phosphate-buffered saline at $-80^{\circ} \mathrm{C}$. RNA was extracted from the cell sediment with a Qiagen RNeasy ${ }^{\circledR}$ kit following the manufacturer's instructions. cfRNA was extracted from urine supernatant by two methods. In method one, microfiltration (MicroF) was used to harvest urine extracellular vesicles (EVs) $[8,15,17]$. Briefly, EVs were filter harvested from $10 \mathrm{ml}$ urine by centrifugation at $4000 \times g$ in a 100-kDa cut-off MicroF device. EV RNA was then extracted using a Qiagen RNeasy kit following the manufacturer's instructions. Method two involved high-volume vacuum extraction (HiVE), which works by drawing large-volume samples over a Norgen RNA-binding column by vacuum using a 25-ml disposable pipette as a large-volume reservoir (see Supplementary data for the complete protocol). Further volumes of urine can be drawn up into the same pipette for extraction on the same RNA-binding column as necessary. The Norgen column binds both large and small RNAs. The small RNA (<150 nt) and large RNA (>150 nt) were separated using a Zymo RNA column (RNA Clean \& Concentrator-5) following the manufacturer's instructions. RNA quantity was assessed using a Qubit 2.0 Fluorometer and Qubit RNA HS Assay. RNA quality was determined using a Bioanalyzer 2100 and the RNA 6000 Pico kit. sRNA yield was assessed with an Agilent sRNA kit. For methodology development, urine samples from three to seven men collected on the same day were mixed together.

\section{RNA amplification \& PCR}

RNA (10-20 ng) was reverse transcribed and amplified using the Ovation Pico WTA System V2 kit (NuGEN, catalog code 3312-48) following the manufacturer's instructions to generate $2-4 \mu \mathrm{g}$ cDNA, which was used to seed PCRs (the PCR primers and conditions are provided in the Supplementary data). Due to the high rate of PCa found in the general population $(\sim 30 \%$ of 30 to 40-year-old men [18]), urine from men proven to be PCa negative was not available. Instead, every set of RT-PCRs contained negative-control tubes consisting of PCR reaction mix to which no cDNA had been added; all of these cDNA-negative controls were negative for PCR product in all cases. PCR product yield was assessed in a semiquantitative manner using Image $\mathrm{J}$ software following the method of Antiabong et al. [19].

\section{RESULTS \& DISCUSSION}

\section{Comparison of cell sediment \& cfRNA}

RNA was extracted from the cell sediment and cell-free supernatant fractions of 201 urine samples. A microfiltration method (see Materials \& methods) was used to harvest $\mathrm{EVs}$ from urine supernatant with a 100-kDa cut-off filter [8]. Both cell sediment and cfRNA were then extracted with an RNeasy kit (Qiagen, Germany). Yields varied widely among samples. Mean cfRNA yields (252 $\mathrm{ng}$ ) were significantly lower than mean cell RNA yields (412 ng; $p<0.001$, Wilcoxon). Pairwise analysis of individual samples demonstrated that there was no evidence of even a weak correlation between cell and cfRNA yields (Pearson $r=0.086)($ Figure 1A).

RNA (10 ng) was amplified prior to TaqMan analysis with a whole transcriptome amplification kit (see Materials \& methods), $10 \mathrm{ng}$ of cDNA was used in each PCR and transcript levels were calculated relative to this input amount. TaqMan quantitative RT-PCR (qRT-PCR) was performed to directly compare the relative amounts of transcript levels of three prostate-related genes in paired-cell and cfRNA samples. These three gene transcripts were selected for their proven usefulness in analyzing urine for the presence of PCa $[11,20,21]: K L K 3$, a prostate-specific transcript that encodes prostate-specific antigen (PSA) $(n=87)$ and two transcripts overexpressed in $\mathrm{PCa}$;
$P C A 3$ (prostate cancer antigen $3 ; \mathrm{n}=84$ ); and OR51E2/PSGR (prostate-specific G-protein coupled receptor; $n=80$ ). Transcript levels in cancer samples were significantly higher in cfRNA compared with cell pellet RNA for $K L K 3$ (>4x; mean $\mathrm{C}_{\mathrm{T}}=25.0$ vs 27.5), OR51E2 $\left(>8 \mathrm{x} ; \mathrm{C}_{\mathrm{T}}=24.9\right.$ vs 28.3$)$ and PCA3 $(32 \mathrm{x}$; $\mathrm{C}_{\mathrm{T}}=21.3$ vs 26.2$)($ all $\mathrm{p}<0.05$, Wilcoxon) (Figure 1B-D).

These results suggest that in general the vast majority of $K L K 3, P C A 3$ and OR51E2 transcripts in whole urine originate in the cell-free EV fraction. PCa cells have been reported to be a small fraction of the many types of cells found in urine which include inflammatory cells, urothelial cells, umbrella cells, squamous cells and mature sperm [22]. In contrast, the majority of EV RNA appears to arise from the prostate [8]. These observations led us to investigate cfRNA biomarkers further.

\section{Variation in RNA yields from post-DRE} urine samples

We examined the urine cfRNA yields extracted by the MicroF method from 315 men after DREs performed by one of six different clinicians. Clinicians were advised to perform three strokes per prostate lobe as recommended for the PCA3 test [11], and urine was then collected 10 min later. RNA yields for all DRE samples varied from 0.1 to $2200 \mathrm{ng}$ (mean, $214 \mathrm{ng}$ ). Some of this variation is associated with the clinician performing the DRE, as median RNA yields varied greater than eightfold among the six clinicians (30-244 ng; $p<0.001$, KruskalWallis) (Figure 1E), suggesting that the DRE technique can influence RNA yield. RNA yields from 14 non-DRE samples collected in the clinic were significantly lower in comparison (range, <1-140 ng; median, $<1 \mathrm{ng}$; $\mathrm{p}<0.001$, Wilcoxon).

\section{Urine preservative, cfRNA yield \& integrity}

MicroF cfRNA yields decreased rapidly when urine was maintained without preservative (Figure 2A \& C) and decreased further with losses of up to $52 \%$ after 6 months of storage at $-80^{\circ} \mathrm{C}$ (Figure $2 \mathrm{~A}$ ). MicroF yields of RNA from seven urine samples stored at $-80^{\circ} \mathrm{C}$ for 3 years were $<1 \mathrm{ng}$ in six of the seven samples (see below and Figure 3 ). 

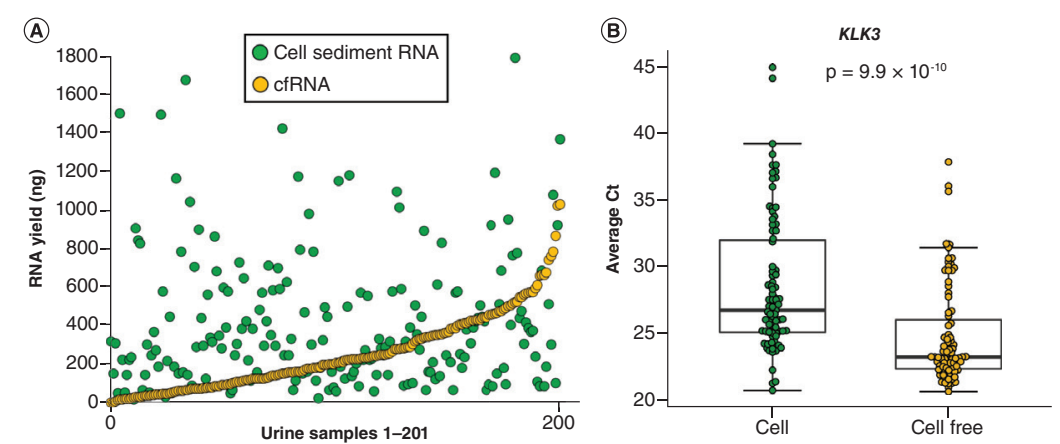

(C)
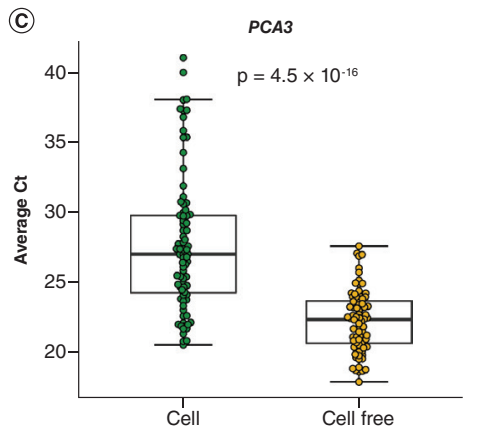

(E)

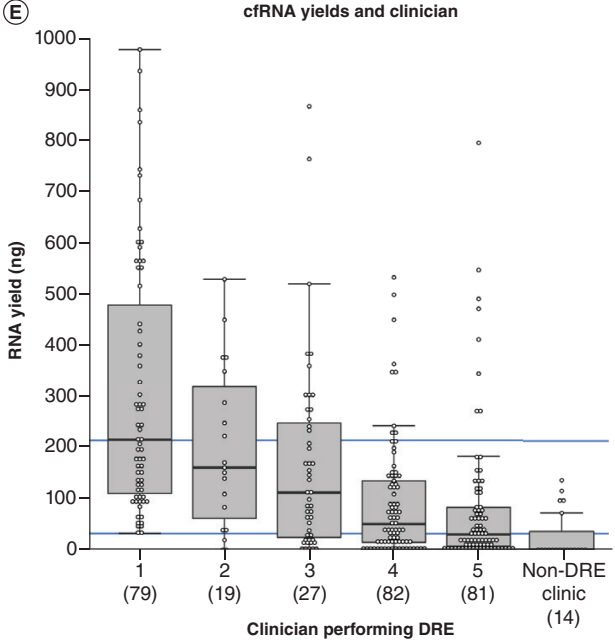

(D)

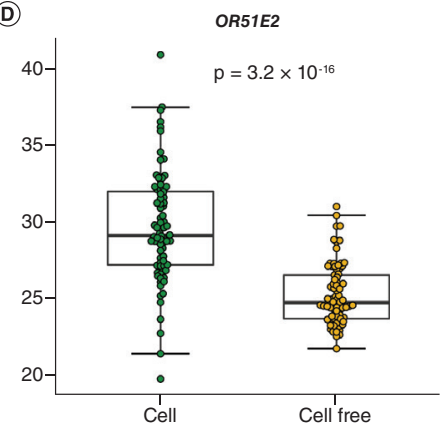

into five aliquots and stored at $-80^{\circ} \mathrm{C}$ for up to 6 months. cfRNA was extracted on the day of urine collection, after 1 week, and after 1 , 3 and 6 months and was assessed by yield (Qubit) and integrity (Bioanalyzer) (Figure 2A \& B). Yields from preserved samples were better than those for the nonpreserved samples, with up to $10 \%$ higher yields even on the day of collection (paired urine sample comparison; $p<0.05$, Wilcoxon). cfRNA yields from non-preserved urine continued to drop over the next 6 months while preserved urine yields stabilized. Yields were similar for both preservatives at 6 months. RNA integrity, as measured by the Bioanalyzer RNA integrity number (RIN), was remarkably stable after 6 months in Norgen preservative and was much better than for the Hologic preservative (example RINs: 6.3 and 4.2, respectively) (Figure 2B). The Norgen preserved urine samples (1:25 dilution) were more economical on freezer space than the Hologic samples (1:1 dilution). The urine standard operating procedure for this study required only a single freeze/thaw cycle. The effects of further freeze/thaw cycles were not tested.

The efficacy of the HiVE method of extraction (see below) for whole urine stored at room temperature up to 1 week with or without Norgen preservative was examined on three samples. At time 0 , $1 \mathrm{~h}$ and 1, 2 and 7 days the cell sediment was removed and cfRNA extracted. The initial rapid drop in cfRNA yield after $1 \mathrm{~h}$ was reduced by the Norgen preservative. Nonpreserved sample cfRNA yields dropped further by $\sim 25 \%$ in the first $48 \mathrm{~h}$ and continued to drop, whereas the preserved sample yields remained stable (Figure 2B \& C). RNA integrity from 7-day-old non-preserved urine was poor compared with the preserved urine (example RINs: 1.8 and 6.1, respectively) (Figure 2D), and the mean yield from preserved urine samples (347 ng) was higher than for non-preserved samples (198.2 ng; $\mathrm{p}=0.1$, Wilcoxon). Preservative manufacturer Norgen states that urine samples remain viable for use for up to 2 years at room temperature.

Two urine preservatives were tested: Hologic, which is used in the PCA3 urine test [11] (1:1 dilution per the manufacturer's instructions), and Norgen (1:25 dilution). The preservatives were initially tested with five urine supernatant samples stored at $-80^{\circ} \mathrm{C}$ and extracted with the MicroF protocol. Samples were divided into three aliquots (no preservative, Norgen preservative or Hologic preservative), which were then subdivided

\section{RNA extraction method by MicroF \& HiVE}

The MicroF method harvests extracellular vesicles by microsieving $[14,15,17]$. We 


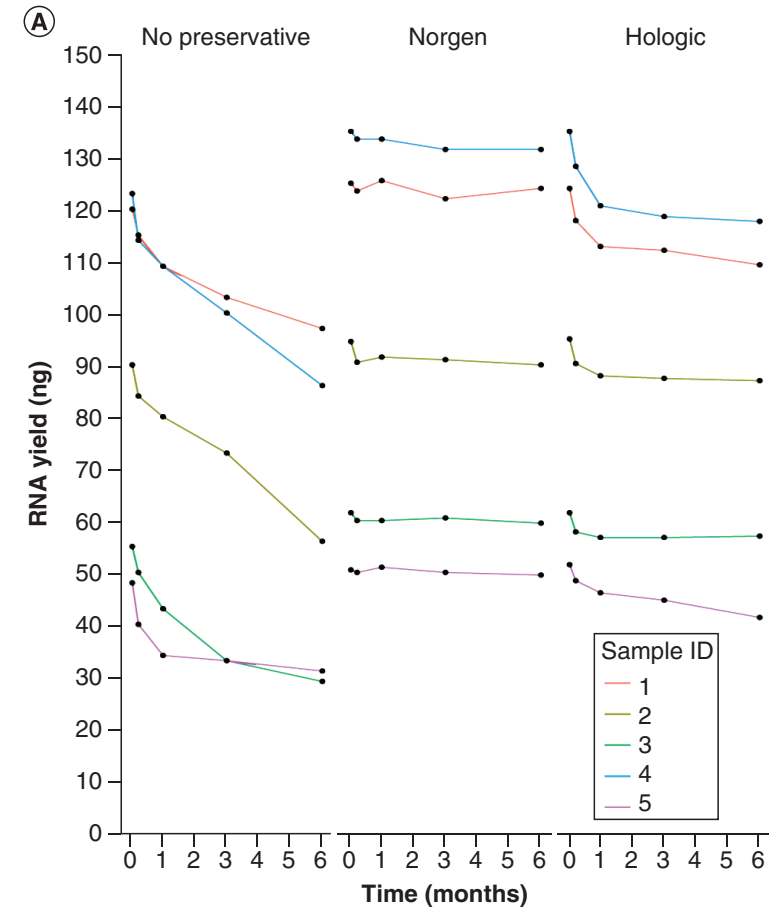

Figure 2. Urine preservative tests (see facing page for parts $C$ and $D$ ). (A) Microfiltration cell-free RNA yields from five urine supernatants stored at $-80^{\circ} \mathrm{C}$ without preservative or with either Norgen or Hologic preservative for up to 6 months. Urine samples were extracted from $2 \mathrm{ml}$ supernatant on the day of collection, after 1 week, and after 1, 3 and 6 months. (B) Bioanalyzer assessment of RNA samples from sample 1 in (A). (C) Highvolume vacuum extraction RNA yields from three whole urine samples stored at room temperature with and without Norgen urine preservative extracted at time 0 , at $1 \mathrm{~h}$ and at 1,2 and 7 days. (D) Bioanalyzer traces for representative samples from (B). Urine was stored at room temperature for 7 days with Norgen preservative (lower panel) and without preservative (upper panel).

(B)
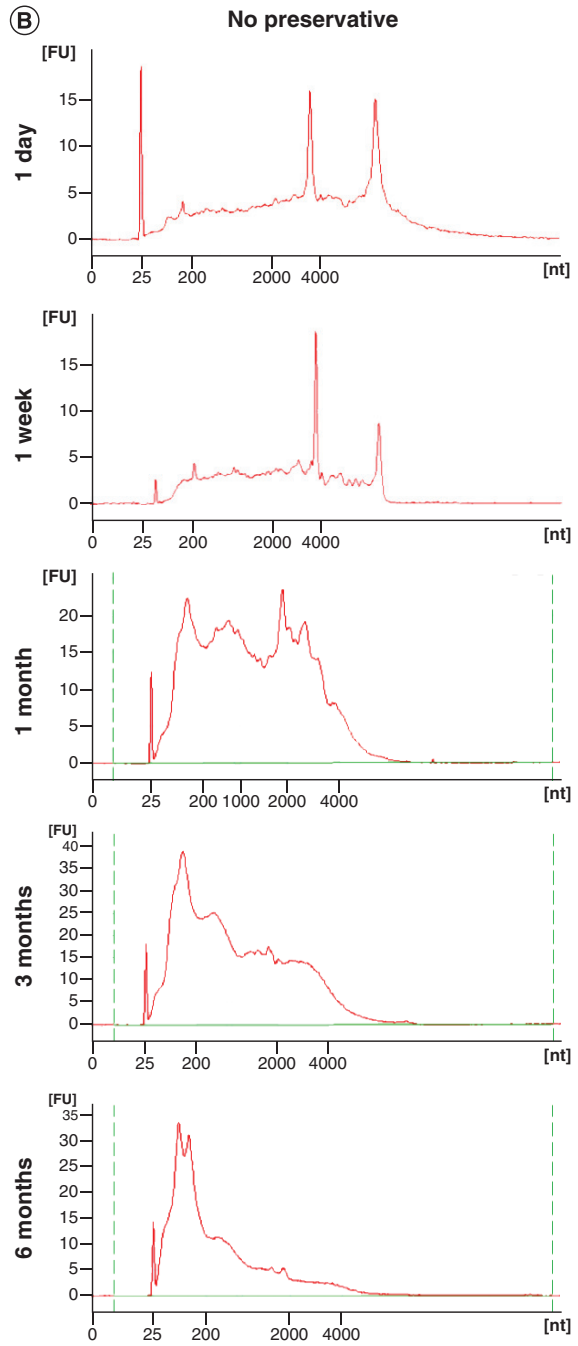
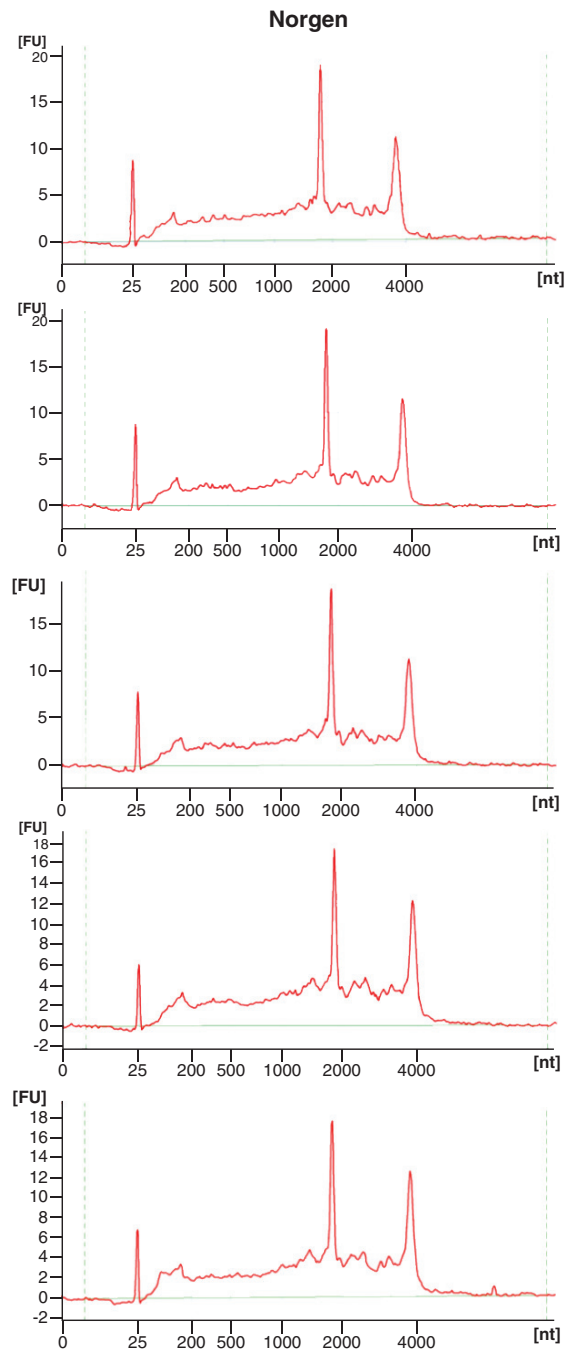

Hologic
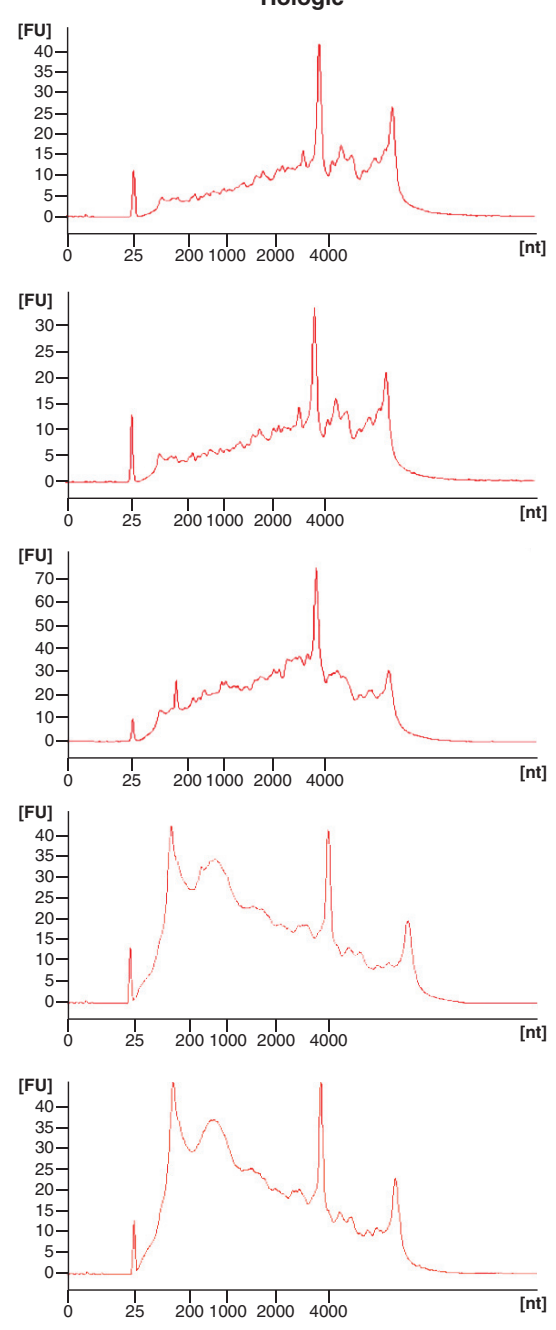


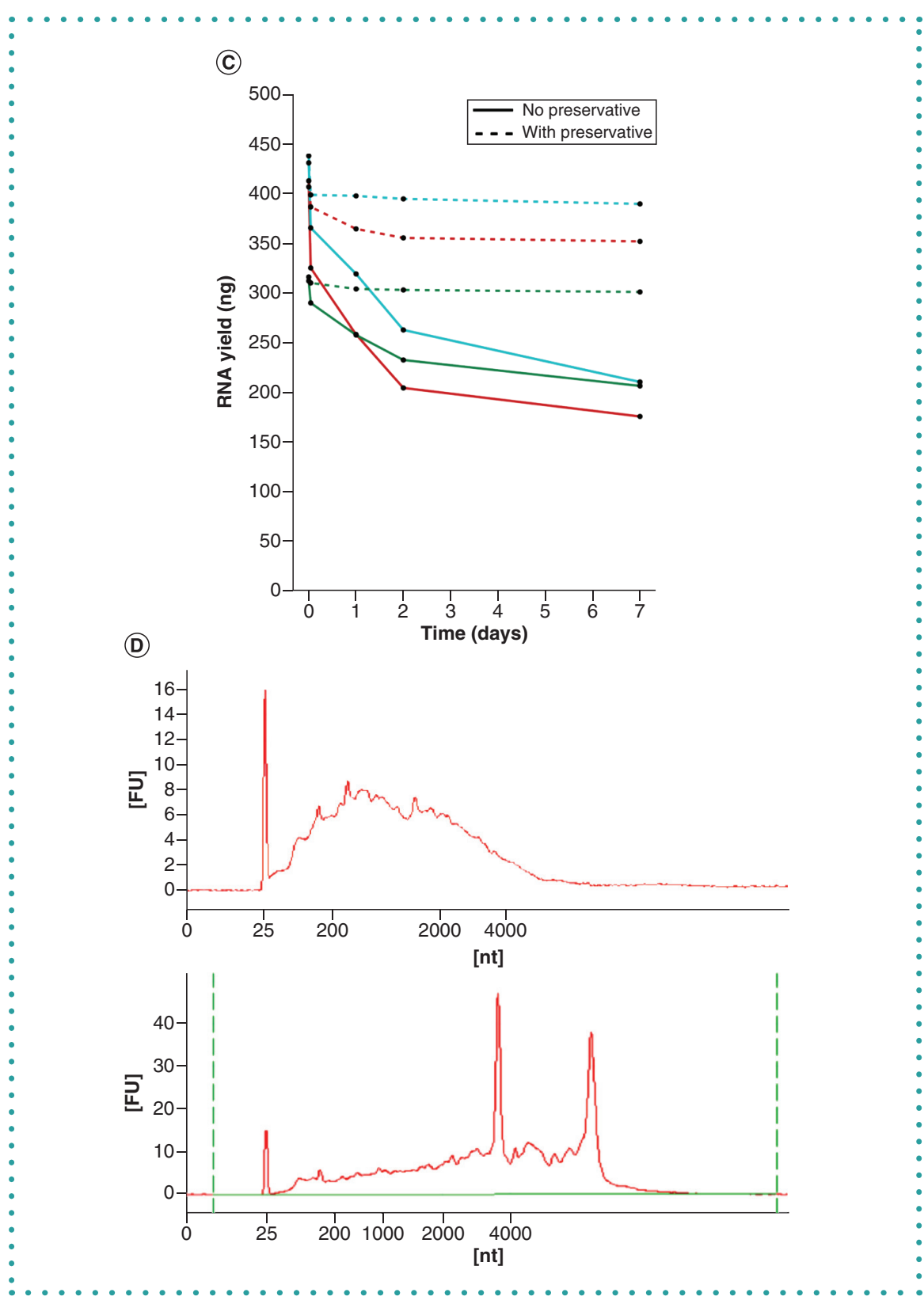

have processed $>900$ samples by this method and have encountered problems with frequent blockage of the expensive microfiltration units and extremely poor yields when extracting cfRNA from historic frozen unpreserved urine (see below and Figure 3A). We examined other published methods of extracting RNA, all of which had some disadvantages (e.g., time consuming, expensive, low input volume and vesicle selection) (see Supplementary Table 1). We therefore set out to devise a simpler and cost-effective means of extracting RNA from large volumes $(\geq 10 \mathrm{ml})$ of urine supernatant without having to harvest the EVs first. HiVE works by using a large-volume reservoir that fits into the top of a standard RNA extraction spin column such as the Qiagen RNeasy column. A $30-\mathrm{ml}$ disposable plastic pipette works well as a reservoir with no leakage problems (see Figure 4 \& Supplementary data for equipment setup). The RNeasy column is positioned on a Qiagen vacuum manifold, and the urine/lysis mixture is pulled through the RNA binding column under vacuum. Further volumes of urine/ lysis binding media can be drawn over the same RNA column if required. HiVE was initially tested with a Qiagen RNeasy column, but subsequently we tested a Zymo RNA column that has a lower elution volume (10 ml instead of $20 \mathrm{ml}$ ) and a Norgen RNA column (silicon carbidebased) that binds a larger size range of RNAs, including small RNAs. Yields for cfRNA were $>1.4$-fold higher $(p=0.0052$, Wilcoxon) when using the Norgen column (Figure 3A). HiVE yields were higher than those for MicroF even after separation of sRNA (Figure 3A). Flow-through times for the Norgen column were much slower ( $30 \mathrm{~min})$ than for Qiagen or Zymo ( 3 min), which may account for some of this increased yield.

The manufacturer's protocol for Norgen column extraction involves multiple repetitive spinning and the re-addition of sample/ reagent mix to the Norgen column, making it incompatible with vacuum extraction. To combine the increased binding capabilities of the Norgen column with the simplicity of the Qiagen method, we tested the Qiagen RNeasy RLT lysis buffer with the Norgen RNA binding column and found that the system worked well. Norgen columns and Qiagen RLT buffer can be purchased as separate items. The total RNA (HiVE-total) was then subdivided into large RNA and small RNA fractions using a Zymo RNA column that only strongly binds RNA over 200 nucleotides. Additional ethanol was then added to the eluate, which was applied to a Zymo column for binding and elution of the small RNA fraction (see Supplementary data for full details). The large and small RNA fractions were referred to as HiVE-LRNA and HiVE-sRNA, respectively. Total RNA from the Norgen column had a tendency to slowly degrade at $-80^{\circ} \mathrm{C}$ over a period of 1 month; however, performing Zymo size separation and cleanup within $24 \mathrm{~h}$ was found to stabilize and maintain RNA integrity.

\section{HiVE \& MicroF cfRNA yields}

cfRNA yields for HiVE and MicroF extraction were directly compared in ten pooled urine samples (urine from 3 to 7 men in each pool) on the day of collection. Median RNA yields for HiVE-total and HiVE-LRNA were 102 and 93 ng, respectively, compared with $57 \mathrm{ng}$ from the MicroF method (see Figure 3Aii). We investigated whether the lower MicroF cfRNA yields were due to cfRNA passing through the 100-kDa microfilter. To this end, we used the HiVE method to extract RNA 

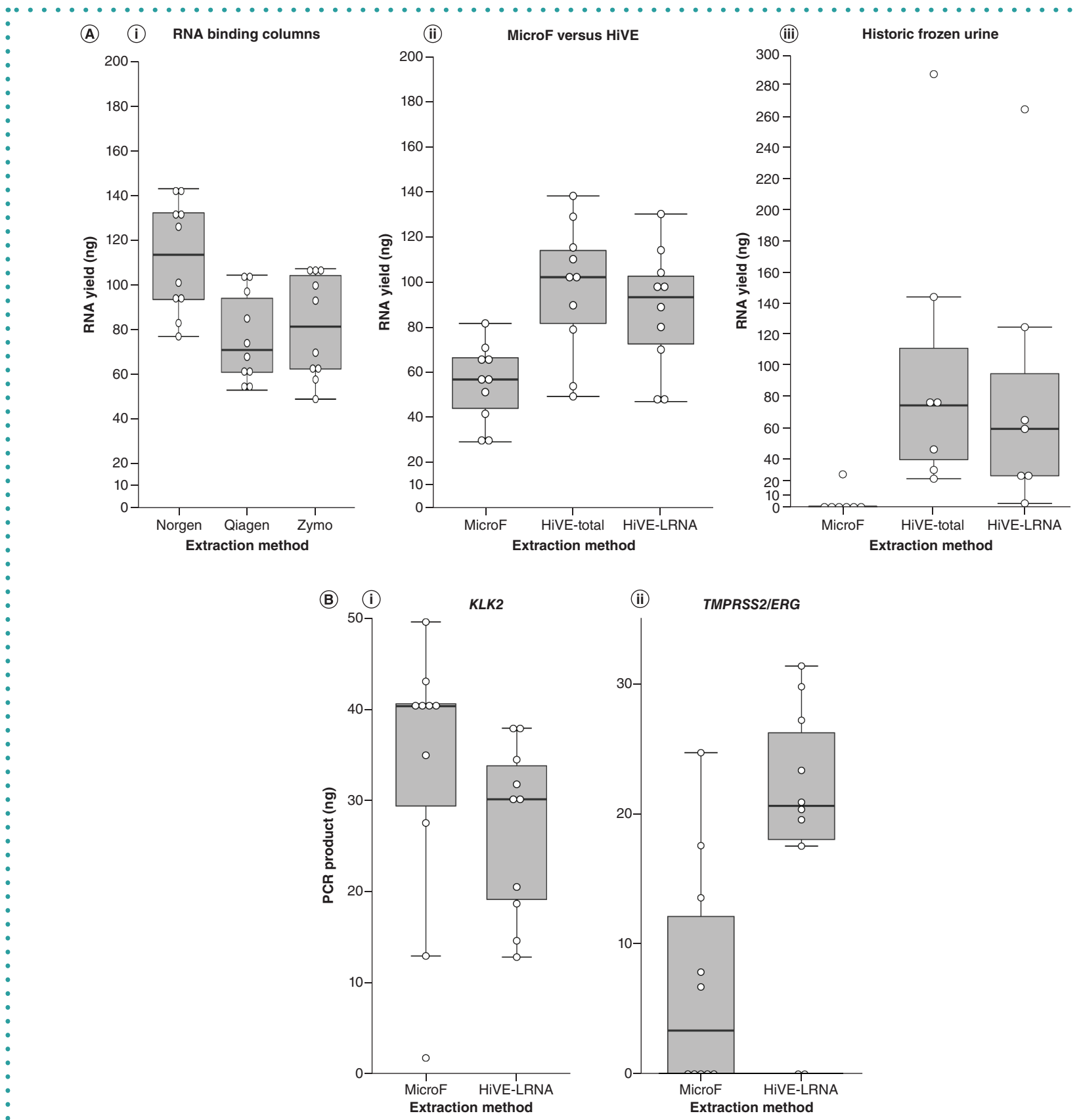

Figure 3. RNA yields and RT-PCR values for microfiltration and high-volume vacuum extraction (HiVE). (A) RNA yields were examined for the following variables: (i) Total RNA yields extracted from $10-\mathrm{ml}$ urine samples $(n=10)$ by HiVE using RNA binding columns from three manufacturers (Norgen, Qiagen and Zymo). (ii) RNA yields from 10 samples extracted on the day of urine collection by MicroF and HiVE (HiVE-total RNA and HiVE-LRNA [ $>200 \mathrm{nt}]$ ). (iii) RNA yields from historic urine samples $(10-\mathrm{ml})$ stored at $-80^{\circ} \mathrm{C}$ without preservative for 3 years $(n=7)$ extracted by both MicroF and HiVE. (B) PCR product yields for KLK2 and TMPRSS2/ERG transcripts in the samples extracted by both MicroF and HiVE. HiVE: High-volume vacuum extraction; LRNA: LargeRNA >200nt; MicroF: Microfiltration.

from the 100-kDa flow-through. Mean total RNA yield was $18 \mathrm{ng}$, accounting for $\sim 35 \%$ of the difference in yields between the MicroF and HiVE total RNA extractions. HiVE-sRNA was in the size range of microRNAs and sRNAs (Supplementary Figure 1). Yields from 10 samples ranged from 0 to $69 \mathrm{ng}$ (mean, $25 \mathrm{ng}$ ). The utility of HiVE to extract cfRNA from historic whole urine frozen without preservative for up to 3 years was investigated. Whole urine samples $(n=7)$ were thawed at $4^{\circ} \mathrm{C}$ for $4 \mathrm{~h}$ and cell sediment removed. cfRNA yields were $\sim 21$-fold higher with HiVE $(p=0.002$, Kruskal-Wallis) (Figure 3A) than with MicroF extraction.

\section{PCR analysis of RNA extracted by MicroF \& HiVE}

Ten urine samples were extracted by both MicroF and HiVE and examined by PCR for levels of three transcripts (see Materials \& methods): KLK2 (prostate-specific), TMPRSS2:ERG fusion gene transcripts (PCa-specific, present in $50 \%$ of $\mathrm{PCa}$ ), and 
PCA3 (overexpressed in PCa). As MicroF cfRNA yields were often $<100 \mathrm{ng}$, all samples used for PCR were first amplified as cDNA using the NuGEN WTA kit [23]. PCR products were quantified by band intensity on agarose gels using Image $J$ software $[19,24,25]$. There was no credible evidence that $K L K 2$ product yields were significantly different (median yields, $30 \mathrm{ng}$ for HiVE vs 40 ng for MicroF; $p=0.089$, Wilcoxon) (Figure 3B). Product yields and the sensitivity of detection of TMPRSS2:ERG were significantly higher for HiVE RNA (8/10 positive) than MicroF RNA (5/10 positive), with median product yields for MicroF being less than a fifth of those for HiVE (3 ng vs $21 \mathrm{ng} ; \mathrm{p}=0.015$, Wilcoxon). The reason for this was hypothesized to be the Millipore $100-k D a$ filter used in the MicroF method having some selective effect on the extracellular vesicles, in contrast to HiVE, which has no prior selection procedure.

\section{At-home collection of non-DRE urine samples for PCa biomarker analysis}

We examined whether the consistency of urine biomarker levels could be improved by collecting urine from patients at home from their first micturition of the day. We implemented the technical improvements entailed above into an at-home collection protocol using Norgen preservative and HiVE extraction (see Figure 5 \& Supplementary data for more information about the at-home collection kit and protocol for urine collection). The 30-ml tubes used for collection contained Norgen preservative that had been dried to the bottom of the tube overnight under vacuum (also available predried from Norgen; see Supplementary data). The dried preservative remained in place at the bottom of the tube during transport to the patient and dissolved over a period of $20 \mathrm{~min}$ after the addition of urine. Capped tubes were placed in a sealable plastic bag with wadding and sent to the laboratory in a leakproof SafeBox container. Study information and two consent forms were provided to the patient in the kit, along with a telephone contact number if further information was required.

Fourteen men were enrolled to provide three urine samples: post-DRE urine in the clinic, non-DRE urine collected from the first micturition of the day at home $(\mathrm{HO})$, and a non-DRE sample collected at home $1 \mathrm{~h}$ later



Figure 4. RNA high-volume vacuum extraction (HiVE). (A) Overview of the vacuum manifold setup for extraction of RNA from urine. (B) Close-up of a $25-\mathrm{ml}$ pipette inserted into the Norgen RNA column; no additional support for the column is required. Urine is slowly pulled through the Norgen column under vacuum ( $30 \mathrm{~min})$.
(H1), which was used to examine how much cfRNA can accumulate in $1 \mathrm{~h}$. Yields of cfRNA were comparable for post-DRE samples and at-home collected samples (median yield comparisons all $p>0.05$, Wilcoxon test) (Figure 6A). PCR was performed for $K L K 2$, PCA3 and TMPRSS2:ERG. In pairwise comparisons of PCR product yields, no statistically significantly differences were found for $K L K 2$ and PCA3, but TMPRSS2:ERG was found to differ significantly between DRE and homecollected samples (DRE vs $\mathrm{HO}, \mathrm{p}=0.009$; DRE vs $\mathrm{H} 1, \mathrm{p}=0.029$; $\mathrm{HO}$ vs $\mathrm{H} 1, \mathrm{p}=0.52$,
Wilcoxon). TMPRSS2:ERG was detectable in eight out of $14 \mathrm{HO}$ and $\mathrm{H} 1$ samples but was only detectable in three out of 14 post-DRE clinic samples ( $p=0.12$; Fisher's exact test) (Figure 6B). We hypothesize that this low rate of detection in DRE samples may be due to earlier urination in the hospital depleting $\mathrm{PCa}$ biomarkers from the urethra prior to urine collection; alternatively, it may imply the presence of anterior tumors.

Although the effect of nycturia on the $\mathrm{HO}$ samples is unknown, it is notable that the rates of detection of $P C A 3$ and TMPRSS2:ERG

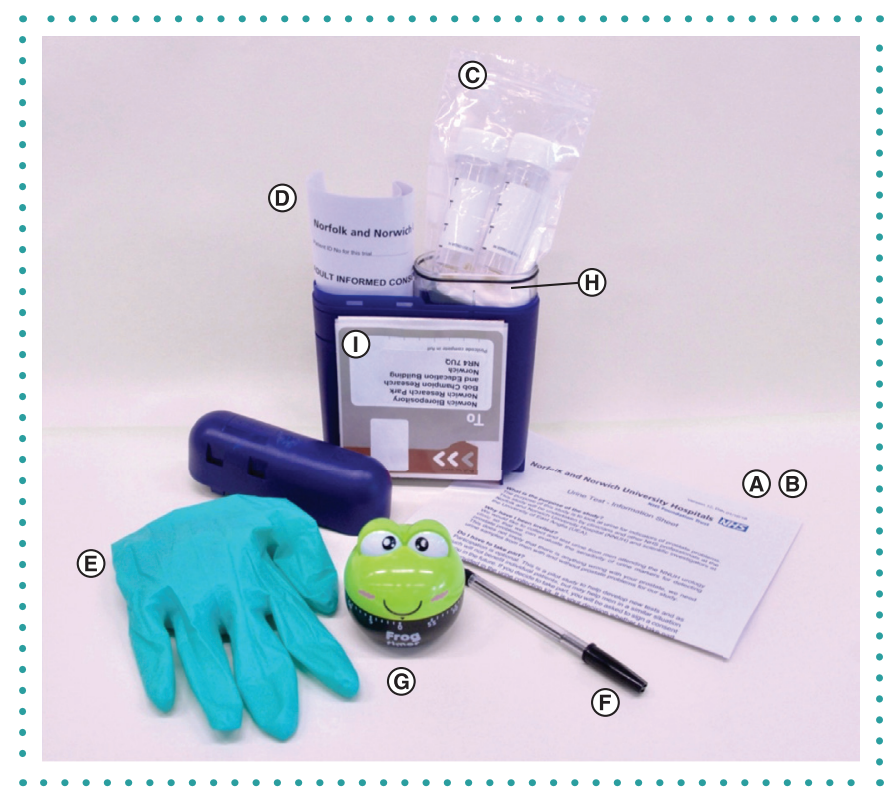

Figure 5. Contents of the at-home collection kit. (A) Invitation to participate. (B) Study information sheet. (C) Two urine collection tubes $(30 \mathrm{ml})$ containing dried Norgen preservative. (D) Two consent forms (one for the patient to keep and the other to return with the samples). (E) Disposable non-allergenic glove. (F) Pen (to write the time and date on the tubes). (G) 1-h frog timer. (H) Sealable plastic bag with wadding. (I) Preaddressed postage-paid SafeBox for returning the samples. 


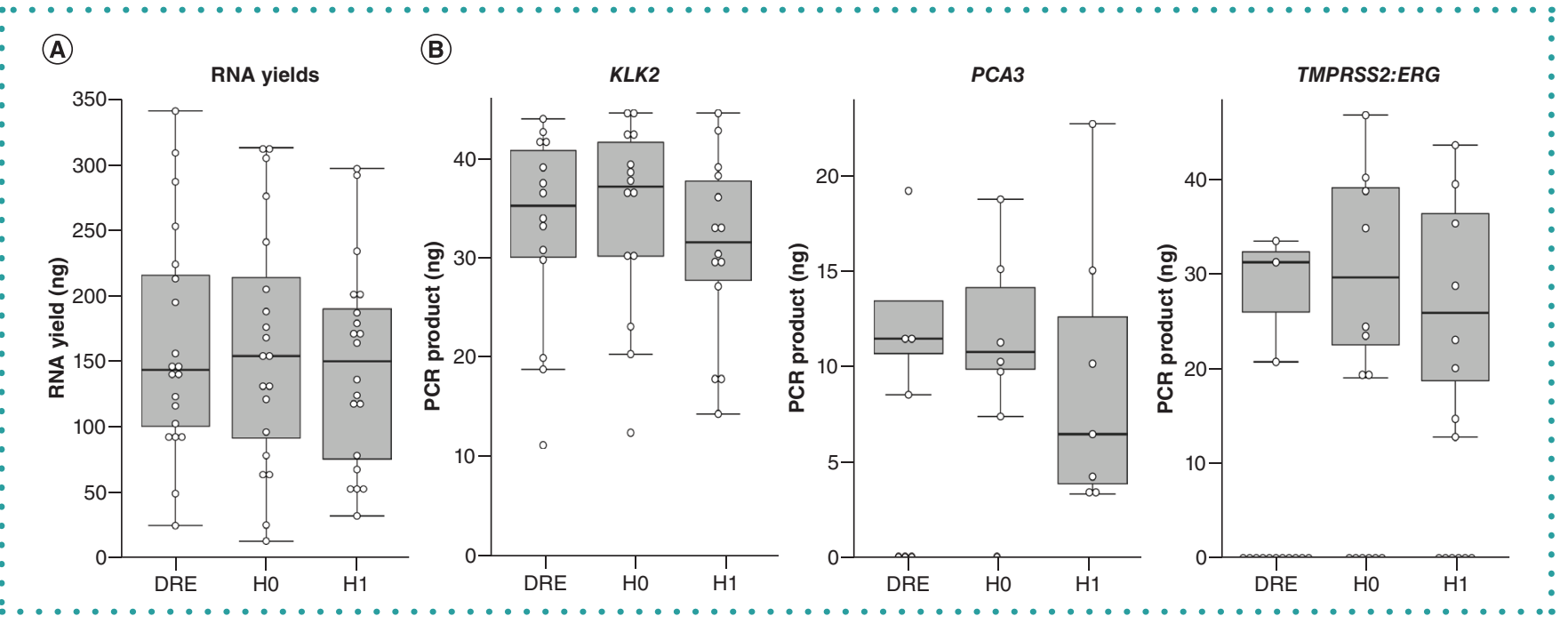

Figure 6. Post-digital rectal examination and at-home collection samples. (A) HiVE-LRNA yields from 20 men from whom three urine samples were collected. DRE indicates urine collected in the clinic post-DRE; H0, urine collected at home first thing in the morning (time 0 ); and $\mathrm{H} 1$, a second urine sample collected at home $1 \mathrm{~h}$ later. RNA was extracted from $10 \mathrm{ml}$ of the $30 \mathrm{ml}$ of urine collected. (B) Home collection samples $(n=14)$ were analyzed by RT-PCR for three transcripts (KLK2, TMPRSS2:ERG and PCA3).

DRE: Digital Rectal Examination; HiVE: High-volume vacuum extraction.

PCR products in $\mathrm{HO}$ samples were higher than in the post-DRE samples. Median levels of PCR products in $\mathrm{HO}$ samples were $11-33 \%$ higher than for samples collected $1 \mathrm{~h}$ later ( $\mathrm{H} 1)$ (Figure 6). From these data, we hypothesize that a minimum of 2 or 3 $h$ could be required for transcript levels to reach those of the $\mathrm{HO}$ samples; therefore, in our letter to the patient, we asked them to provide a urine sample after a minimum of $3 \mathrm{~h}$ of sleep. Ejaculation is expected to deplete prostate biomarkers and may have other unknown effects on the prostate urine risk test. For this reason, it may be necessary to request that the patient wait $24 \mathrm{~h}$ after sexual activity before providing a sample.

\section{FUTURE PERSPECTIVE}

The methodology and at-home collection protocol in this paper offer a simple and cost-effective means of testing and monitoring men for PCa. We intend to use this methodology to implement a clinical test for aggressive PCa within the next 10 years. The collection of urine from men at home avoids the randomness of DRE and can increase the levels of PCa biomarkers in the urine. Connell et al. [8] have shown that an elevated 'prostate urine risk' signature can be used to identify aggressive $\mathrm{PCa}$, thus avoiding overdiagnosis and identification of indolent disease. The at-home collection protocol could revolu- tionize how men with PCa on active surveillance are monitored for disease progression, as men would have to visit the clinic only for a positive urine result, which is preferable to the current situation where they are recalled to the clinic every $6-12$ months for PSA testing and painful and expensive biopsies. A negative result for the prostate urine risk signature test could allow men to be retested only every $2-3$ years, reducing both patient stress and hospital workloads. This protocol will also enable screening for other urinary cancers such as bladder and kidney.

\section{AUTHOR CONTRIBUTIONS}

J Clark, C Cooper and M Webb conceived the study. M Webb performed the underlying research for the study. J Clark, R Hurst, M Olivan, I Guldvik, M Webb and I Mills designed and performed the cell versus cfRNA comparison and analysis. M Webb, J Clark, $\mathrm{R}$ Mills and $\mathrm{K}$ Manley set up the home collection study. M Webb, D Brewer and S Connell analyzed the data. J Clark and $M$ Webb wrote the manuscript. D Brewer, C Cooper and S Connell critically revised the manuscript.

\section{FINANCIAL \& COMPETING INTERESTS DISCLOSURE}

This study was funded by the following charitable organizations: Movember Foundation GAP1 Urine Biomarker project,
Masonic Charitable Foundation, Bob Champion Cancer Trust, King family, Andy Ripley Memorial Fund, and Stephen Hargrave Trust. The authors have no other relevant affiliations or financial involvement with any organization or entity with a financial interest in or financial conflict with the subject matter or materials discussed in the manuscript apart from those disclosed.

No writing assistance was utilized in the production of this manuscript.

\section{ETHICAL CONDUCT OF RESEARCH}

Ethical approval was gained for the collection and analysis of patient urine samples from the East of England Research Ethics Committee, UK (IRAS project ID 96199, REC reference 12/EE/0058). Informed consent was obtained from all of the participants involved.

\section{OPEN ACCESS}

This work is licensed under the AttributionNonCommercial-NoDerivatives 4.0 Unported License. To view a copy of this license, visit http://creativecommons.org/licenses/ by-nc-nd/4.0/

\section{SUPPLEMENTARY DATA}

To view the supplementary data that accompany this paper please visit the journal website at: www.future-science. com/doi/suppl/10.2144/btn-2019-0092 


\section{REFERENCES}

1. Zlotta AR, Egawa S, Pushkar D et al. Prevalence of prostate cancer on autopsy: cross-sectional study on unscreened Caucasian and Asian men. J. Natl Cance Inst. 105(14), 1050-1058 (2013).

2. Bray F, Ferlay I, Soerjomataram I et al. Global Cancer Statistics 2018: GLOBOCAN estimates of incidence and mortality worldwide for 36 cancers in 185 countries. CA Cancer J. Clin. 68(6), 394-424 (2018).

3. Arora R, Koch MO, Eble JN, Ulbright TM, Li L, Cheng L. Heterogeneity of Gleason grade in multifocal adenocarcinoma of the prostate. Cancer 100(11), 2362--2366 (2004).

4. Cheng L, Poulos CK, Pan C-X et al. Preoperative prediction of small volume cancer (less than $0.5 \mathrm{ml}$ ) in radica prostatectomy specimens. J. Urol. 174(3), 898-902 (2005)

5. Bastacky SI, Wojno KJ, Walsh PC, Carmichael MJ, Epstein JI. Pathological features of hereditary prostate cancer. J. Urol. 153(3 Pt. 2), 987-992 (1995).

6. Isaacs JT. Prostatic structure and function in relation to the etiology of prostatic cancer. Prostate 4(4), 351-366 (1983).

7. Duijvesz D, Luider T, Bangma $\mathrm{CH}$, Jenster $\mathrm{G}$. Exosomes as biomarker treasure chests for prostate cancer. Eur. Urol. 59(5), 823-831 (2011).

8. Connell SP, Hanna M, McCarthy F et al. A four-group urine risk classifier for predicting outcome in prostate cancer patients. BJU Int. doi:10.1111/bju. 14811 (2019) (Epub ahead of print).

9. Tomlins SA, Alshalalfa M, Davicioni E et al. Characterization of 1,577 primary prostate cancers reveals novel biological and clinicopathological insights into molecular subtypes. Eur. Urol. 68(4), 555-567 (2015).

10. Van Neste L, Hendriks RJ, Dijkstra S et al. Detection of high-grade prostate cancer using a urinary molecular biomarker-based risk score. Eur. Urol. 70(5), 740-748 (2016).

11. Groskopf J, Aubin SMJ, Deras IL et al. APTIMA PCA3 molecular urine test: development of a method to aid in the diagnosis of prostate cancer. Clin. Chem. 52(6), 1089-1095 (2006).

12. Roehrborn CG, Girman CJ, Rhodes T et al. Correlation between prostate size estimated by digital rectal examination and measured by transrectal ultrasound. Urology 49(4), 548-557 (1997).

13. Dubé CE, Fuller BK, Rosen RK, Fagan M, O'Donnell $\mathrm{J}$. Men's experiences of physical exams and cancer screening tests: a qualitative study. Prev. Med. 40(6), 628-635 (2005).

14. McKiernan J, Donovan MJ, O'Neill V et al. A novel urine exosome gene expression assay to predict high-grade prostate cancer at initial biopsy. JAMA Oncol. 2(7), 882-889 (2016)

15. Pellegrini KL, Patil D, Douglas KJS et al. Detection of prostate cancer-specific transcripts in extracellular prostate cancer-specific transcripts in extracellular
vesicles isolated from post-DRE urine. Prostate $77(9)$, v90-999 (2017)

16. Gupta S, Halm EA, Rockey DC et al. Comparative effectiveness of fecal immunochemical test outreach, colonoscopy outreach, and usual care for boosting colorectal cancer screening among the underserved JAMA Intern. Med. 118(24), 6072-6078 (2013).

17. Miranda KC, Bond DT, McKee $M$ et al. Nucleic acids within urinary exosomes/microvesicles are potential biomarkers for renal disease. Kidney Int. 78(2), 191-199 (2010).
18. Sakr WA, Grignon DJ, Crissman JD et al. High grade prostatic intraepithelial neoplasia (HGPIN) and prostatic adenocarcinoma between the ages of 20-69: an autopsy study of 249 cases. In Vivo 7(3), 439-443 (1994).

19. Antiabong JF, Ngoepe MG, Abechi AS. Semi-quantitative digital analysis of polymerase chain reaction- electrophoresis gel: potential applications in low-income veterinary laboratories. Vet. World 9, 935-939 (2016).

20. Rodriguez M, Siwko S, Liu M. Prostate-specific G-protein coupled receptor, an emerging biomarker regulating inflammation and prostate cancer invasion. Curr. Mol. Med. 16(6), 526-532 (2016).

21. Sequeiros T, Bastarós JM, Sánchez $M$ et al. Urinary biomarkers for the detection of prostate cancer in patients with high-grade prostatic intraepithelial neoplasia. Prostate 75, 1102-1113 (2015).

22. Fujita K, Pavlovich CP, Netto GJ et al. Specific detection of prostate cancer cells in urine by multiplex immunofluorescence cytology. Hum. Pathol. 40(7), 924-933 (2009).

23. Faherty SL, Campbell CR, Larsen PA, Yoder AD. Evaluating whole transcriptome amplification for gene profiling experiments using RNA-Seq. BMC Biotechnol. 15(1), 17-19 (2015).

24. Schneider CA, Rasband WS, Eliceiri KW. NIH Image to ImageJ: 25 years of image analysis. Nat. Methods 9(7), 671-675 (2012)

25. Schindelin J, Arganda-Carreras I, Frise E et al. Fiji: an open-source platform for biological-image analysis. Nat. Methods 9(7), 676-682 (2012). 